\title{
Practical Stability in Terms of Two Measures for Hybrid Dynamic Systems \\ by
}

\author{
Shurong SUN, Zhenlai HAN, Elvan AKIN-BOHNER and Ping ZHAO \\ Presented by Czesław OLECH
}

Summary. We study hybrid dynamic systems on time scales. Using Lyapunov-like functions, we obtain sufficient conditions for practical stability and strict practical stability in terms of two measures for hybrid dynamic systems on time scales.

1. Introduction. In this paper, we present some sufficient conditions for practical stability and strict practical stability in terms of two measures for hybrid dynamic systems on time scales by using Lyapunov-like functions. The practical stability problem, introduced by LaSalle and Lefschetz [12], deals with the question of whether the system state evolves within certain subsets of the state-space. This is very useful in estimating the worst-case transient and steady-state responses and in verifying pointwise in time constraints imposed on the state trajectories. Thus, practical stability is concerned with quantitative analysis as opposed to Lyapunov analysis which is qualitative in nature. Lyapunov analysis may not always be useful in an engineering sense. For instance, an equilibrium point may not be stable in the sense of Lyapunov and yet the system response may be acceptable in the vicinity of this equilibrium; an example involving a van der Pol oscillator is given in [12]. On the other hand, an equilibrium point may be stable in the sense of Lyapunov, but the domain of stability (see [5] for its definition) could be so small as to render the system practically unstable. A striking example of this phenomenon is the Reynolds system used to study the flow of water along a tube of circular cross-section at various speeds [1]. Laminar

2010 Mathematics Subject Classification: 34N05, 34A38, 34D20.

Key words and phrases: hybrid systems on time scales, variational Lyapunov method, $\left(h_{0}, h\right)$-practical stability. 
flow, i.e. smooth flow of water particles parallel to the axis, is stable at all speeds. However, the domain of stability at very high flowrates is so absurdly small as to render laminar flow practically unstable.

The study of hybrid systems [13] is caused by modeling, design and validation of interacting systems of continuous processes and computer programs. Therefore, the identifying characteristic of hybrid systems is that they incorporate both continuous components, usually called plants, which are governed by differential equations, and also digital components such as digital computers, sensors and actuators controlled by programs. Moreover, the growing demand for control systems that are capable of controlling complex nonlinear continuous plants with discrete intelligent controllers can be addressed by the method of hybrid systems [4].

In general, a hybrid dynamic system is a system with different kinds of time dynamics, e.g., continuous, discrete, or impulsive, in different interacting parts of the system. Recently, the theory of dynamic systems on time scales has gained impetus since it demonstrates the interplay of two different theories, namely, the theories of continuous and discrete dynamic systems [6, 2, 3, 9,

It is important to note that dynamic systems on time scales include hybrid systems in general. In the stability and practical stability aspect, Lakshmikantham and Vatsala [10] introduced hybrid dynamic systems on time scales and obtained the practical stability for such systems. P. G. Wang, Liu, $\mathrm{Wu}$ and $\mathrm{Wu}[16,14,15]$ studied the stability criteria in terms of two measures for discrete systems and the practical stability of impulsive hybrid differential systems in terms of two measures on time scales. Lakshmikantham and Mohapatra [8] advanced the concept of strict stability for differential systems, and Lakshmikantham and Zhang [11] developed the idea of strict practical stability of delay differential systems. For all kinds of practical stability for differential systems we refer to [7].

The main purpose of this paper is to establish criteria for practical stability and strict practical stability in terms of two measures for hybrid dynamic systems on time scales. Some ideas in this paper are motivated by [14 16], and some results are extensions of those in [16] for discrete hybrid systems.

2. Preliminaries. Let $\mathbb{T}$ be a time scale (a closed nonempty subset of $\mathbb{R})$. On $\mathbb{T}$ we define the forward and backward jump operators by

$$
\sigma(t):=\inf \{s \in \mathbb{T} \mid s>t\} \quad \text { and } \quad \rho(t):=\sup \{s \in \mathbb{T} \mid s<t\} .
$$

A point $t \in \mathbb{T}$ is said to be left-dense if $\rho(t)=t$, right-dense if $\sigma(t)=t$, left-scattered if $\rho(t)<t$, and right-scattered if $\sigma(t)>t$. The graininess $\mu$ of the time scale is defined by $\mu(t):=\sigma(t)-t$. 
For a function $f: \mathbb{T} \rightarrow \mathbb{R}$ the delta derivative $f^{\Delta}(t)$ at $t \in \mathbb{T}$ is defined to be the number (provided it exists) with the property that for every $\varepsilon>0$, there exists a neighborhood $U$ of $t$ with

$$
\left|f(\sigma(t))-f(s)-f^{\Delta}(t)(\sigma(t)-s)\right| \leq \varepsilon|\sigma(t)-s| \quad \text { for all } s \in U .
$$

A function $f: \mathbb{T} \rightarrow \mathbb{R}$ is said to be rd-continuous if it is continuous at each right-dense point and if there exists a finite left-sided limit at all left-dense points. The set of rd-continuous functions $f: \mathbb{T} \rightarrow \mathbb{R}$ is denoted by $\mathrm{C}_{\mathrm{rd}}(\mathbb{T}, \mathbb{R})$.

Definition 2.1. For each $t \in \mathbb{T}$, let $\mathcal{N}$ be a neighborhood of $t$. Then we define the lower right Dini derivative $D_{+} u^{\Delta}(t)$ by the condition: for given $\varepsilon>0$, there exists a right neighborhood $\mathcal{N}_{\varepsilon} \subset \mathcal{N}$ of $t$ such that

$$
\frac{u(\sigma(t))-u(s)}{\mu^{*}(t, s)}>D_{+} u^{\Delta}(t)-\varepsilon \quad \text { for } s \in \mathcal{N}_{\varepsilon}, s>t,
$$

where $\mu^{*}(t, s) \equiv \sigma(t)-s$.

In case $t$ is right-scattered and $u$ is continuous at $t$, we have as in the case of the derivative

$$
D_{+} u^{\Delta}(t)=\frac{u(\sigma(t))-u(t)}{\mu(t)}=u^{\Delta}(t) .
$$

Definition $2.2([10])$. For each $t \in \mathbb{T}$, let $\mathcal{N}$ be a neighborhood of $t$. Then we define the upper right Dini derivative $D^{+} u^{\Delta}(t)$ by the condition: for given $\varepsilon>0$, there exists a right neighborhood $\mathcal{N}_{\varepsilon} \subset \mathcal{N}$ of $t$ such that

$$
\frac{u(\sigma(t))-u(s)}{\mu^{*}(t, s)}<D^{+} u^{\Delta}(t)+\varepsilon \quad \text { for } s \in \mathcal{N}_{\varepsilon}, s>t
$$

In case $t$ is right-scattered and $u$ is continuous at $t$, we have as in the case of the derivative

$$
D^{+} u^{\Delta}(t)=\frac{u(\sigma(t))-u(t)}{\mu(t)}=u^{\Delta}(t)
$$

Definition 2.3. A function $f(u, v)$ is said to be quasimonotone nondecreasing (respectively, nonincreasing) if for fixed $u$ (or $v), f$ is nondecreasing (respectively, nonincreasing) in $v$ (or $u$ ).

We now state a result on existence of extremal solutions of the initial value problem for the dynamic system

$$
u^{\Delta}=g(t, u), \quad u\left(t_{0}\right)=u_{0},
$$

where $g \in \mathrm{C}_{\mathrm{rd}}\left[I_{0} \times B, \mathbb{R}^{n}\right]$ is such that $\|g(t, u)\| \leq M,(t, u) \in I_{0} \times B$, $I_{0}=\left[t_{0} \leq t \leq t_{0}+a\right] \cap \mathbb{T}, B=\left\{u \in \mathbb{R}^{n}:\left|u-u_{0}\right| \leq b\right\}$, and $M$ is a constant. $\|\cdot\|$ denotes the Euclidean norm in $\mathbb{R}^{n}$. 
Lemma 2.1 ([9, Theorem 2.3.1]). Assume that $g(t, u)$ is quasimonotone nondecreasing in $u$ and for each $i, 1 \leq i \leq n, g_{i}(t, u) \mu(t)+u_{i}$ is nondecreasing in $u_{i}$ for each $t \in \mathbb{T}$. Then there exist minimal and maximal solutions of (2.1) on $\left[t_{0}, t_{0}+\eta\right] \cap \mathbb{T}$, where $\eta=\min (a, b /(2 M+b))$.

We now state a variational comparison result.

Lemma 2.2 ([9, Theorem 2.4.1]). Let all the assumptions of Lemma 2.1 hold and let $m: I \equiv\left[t_{0}, t_{0}+a\right) \cap \mathbb{T} \rightarrow \mathbb{R}^{n}$ be differentiable for each $t \in I$ and satisfy $m^{\Delta}(t) \leq g(t, m(t)), t \in I$. Then $m(t) \leq \beta(t), t \in I$, where $\beta(t)$ is the maximal solution of 2.1.

REMARK 2.1. Lemma2.2 is also valid with $m^{\Delta}(t)$ replaced by $D^{+} m^{\Delta}(t)$.

LEMma 2.3. Let all the assumptions of Lemma 2.1 hold and let $m: I \equiv$ $\left[t_{0}, t_{0}+a\right) \cap \mathbb{T} \rightarrow \mathbb{R}^{n}$ be differentiable for each $t \in I$ and satisfy $m^{\Delta}(t) \geq$ $g(t, m(t)), t \in I$. Then $m(t) \geq r(t), t \in I$, where $r(t)$ is the minimal solution of 2.1.

Proof. The proof is similar to that of Theorem 2.4.1 in [9], and so we omit it here.

REMARK 2.2. Lemma 2.3 is also valid when $m^{\Delta}(t)$ is replaced by $D_{+} m^{\Delta}(t)$.

3. Comparison theorems. We consider the dynamic system

$$
x^{\Delta}=f(t, x), \quad x\left(t_{0}\right)=x_{0},
$$

on $\mathbb{T}$, where $f \in \mathrm{C}_{\mathrm{rd}}\left[\mathbb{T} \times \mathbb{R}^{n}, \mathbb{R}^{n}\right], t_{0} \in \mathbb{T}$, and $x_{0} \in \mathbb{R}^{n}$. We assume, for convenience, that the solution $x(t)=x\left(t, t_{0}, x_{0}\right)$ of (3.1) exists and is unique for $t \geq t_{0}$ on $\mathbb{T}$.

Following Definition 2.1 , we define $D_{+} V^{\Delta}(t, x(t))$ for $V \in \mathrm{C}_{\mathrm{rd}}\left[\mathbb{T} \times \mathbb{R}^{n}, \mathbb{R}^{+}\right]$ by the condition: for given $\varepsilon>0$, there exists a right neighborhood $\mathcal{N}_{\varepsilon} \subset \mathcal{N}$ of $t$ such that

$\frac{1}{\mu(t, s)}[V(\sigma(t), x(\sigma(t)))-V(s, x(\sigma(t)))-\mu(t, s) f(t, x(t))]>D_{+} V^{\Delta}(t, x(t))-\varepsilon$ for $s \in \mathcal{N}_{\varepsilon}, s>t$. As before, if $t$ is right-scattered and $V(t, x(t))$ is continuous at $t$, this reduces to

$$
D_{+} V^{\Delta}(t, x(t))=\frac{V(\sigma(t), x(\sigma(t)))-V(t, x(t))}{\mu(t)}=V^{\Delta}(t, x(t)) .
$$

Following Definition 2.2 , we define $D^{+} V^{\Delta}(t, x(t))$ for $V \in \mathrm{C}_{\mathrm{rd}}\left[\mathbb{T} \times \mathbb{R}^{n}, \mathbb{R}+\right]$ by the condition: for given $\varepsilon>0$, there exists a right neighborhood $\mathcal{N}_{\varepsilon} \subset \mathcal{N}$ of $t$ such that $\frac{1}{\mu(t, s)}[V(\sigma(t), x(\sigma(t)))-V(s, x(\sigma(t)))-\mu(t, s) f(t, x(t))]<D^{+} V^{\Delta}(t, x(t))+\varepsilon$ 
for $s \in \mathcal{N}_{\varepsilon}, s>t$. As before, if $t$ is right-scattered and $V(t, x(t))$ is continuous at $t$, this reduces to

$$
D^{+} V^{\Delta}(t, x(t))=\frac{V(\sigma(t), x(\sigma(t)))-V(t, x(t))}{\mu(t)}=V^{\Delta}(t, x(t)) .
$$

From Lemmas 2.2 and 2.3 , it is now easy to get necessary results in terms of Lyapunov-like functions.

Lemma 3.1 ([9, Th. 3.1.2]). Assume that the solution $x\left(t, t_{0}, x_{0}\right)$ of (3.1) is rd-continuous with respect to the initial data and $\left\|x\left(t, t_{0}, x_{0}\right)\right\|$ is locally Lipschitzian in $x_{0}$. Suppose that

(i) for $V \in \mathrm{C}_{\mathrm{rd}}\left[\mathbb{T} \times \mathbb{R}^{n}, \mathbb{R}^{+}\right], V(t, x)$ is locally Lipschitzian in $x$ for each right-dense point $t \in \mathbb{T}$, and for $t_{0}<s \leq t, z \in \mathbb{R}^{n}$,

$$
D^{+} V^{\Delta}(s, x(t, s, z)) \leq G(s, V(s, x(t, s, z)))
$$

(ii) for $G \in \mathrm{C}_{\mathrm{rd}}\left[\mathbb{T}^{k} \times \mathbb{R}^{+}, \mathbb{R}^{+}\right], G(t, u) \mu(t)+u$ is nondecreasing in $u$ for each $t \in \mathbb{T}$, and the maximal solution $r(t)=r\left(t, t_{0}, u_{0}\right)$ of $u^{\Delta}=$ $G(t, u), u\left(t_{0}\right)=u_{0} \geq 0$, exists for $t \in \mathbb{T}$.

Then if $y\left(t, t_{0}, x_{0}\right)$ is any solution of

$$
y^{\Delta}=G(t, y), \quad y\left(t_{0}\right)=x_{0},
$$

we have

$$
V\left(t, y\left(t, t_{0}, x_{0}\right)\right) \leq r\left(t, t_{0}, V\left(t_{0}, x\left(t, t_{0}, x_{0}\right)\right)\right), \quad t \in \mathbb{T},
$$

provided $V\left(t_{0}, x\left(t, t_{0}, x_{0}\right)\right) \leq u_{0}$.

LEMMA 3.2. Assume that the solution $x\left(t, t_{0}, x_{0}\right)$ of (3.1) is rd-continuous with respect to the initial data and $\left\|x\left(t, t_{0}, x_{0}\right)\right\|$ is locally Lipschitzian in $x_{0}$. Suppose that

(i) for $V \in C_{\mathrm{rd}}\left[\mathbb{T} \times \mathbb{R}^{n}, \mathbb{R}^{+}\right], V(t, x)$ is locally Lipschitzian in $x$ for each $t \in \mathbb{T}$ which is right-dense, and for $t_{0}<s \leq t, z \in \mathbb{R}^{n}$,

$$
D_{+} V^{\Delta}(s, x(t, s, z)) \geq G(s, V(s, x(t, s, z))) ;
$$

(ii) for $G \in C_{\mathrm{rd}}\left[\mathbb{T} \times \mathbb{R}^{+}, \mathbb{R}^{+}\right], G(t, u) \mu(t)+u$ is nondecreasing in $u$ for each $t \in \mathbb{T}$, and the minimal solution $r(t)=r\left(t, t_{0}, u_{0}\right)$ of $u^{\Delta}=$ $G(t, u), u\left(t_{0}\right)=u_{0} \geq 0$, exists for $t \in \mathbb{T}$.

Then if $y\left(t, t_{0}, x_{0}\right)$ is any solution of

$$
y^{\Delta}(t)=G(t, y), \quad y\left(t_{0}\right)=x_{0},
$$

we have

$$
V\left(t, y\left(t, t_{0}, x_{0}\right)\right) \geq r\left(t, t_{0}, V\left(t_{0}, x\left(t, t_{0}, x_{0}\right)\right)\right), \quad t \in \mathbb{T},
$$

provided $V\left(t_{0}, x\left(t, t_{0}, x_{0}\right)\right) \geq u_{0}$. 
Proof. The proof is similar to that of Theorem 3.1.2 in [9], and so we omit it here.

We now consider the hybrid dynamic system

$$
\begin{aligned}
& x^{\Delta}(t)=f\left(t, x, \lambda_{k}\left(\tau_{k}, z_{k}\right)\right), \quad t \in\left[\tau_{k}, \tau_{k+1}\right], \\
& x\left(\tau_{k}\right)=z_{k} \in \mathbb{R}^{n}, \quad k=0,1,2, \ldots,
\end{aligned}
$$

and the perturbed hybrid dynamic system

$$
\begin{aligned}
& y^{\Delta}=g\left(t, y, \lambda_{k}\left(\tau_{k}, z_{k}\right)\right), \quad t \in\left[\tau_{k}, \tau_{k+1}\right], \\
& y\left(\tau_{k}\right)=z_{k} \in \mathbb{R}^{n}, \quad k=0,1,2, \ldots,
\end{aligned}
$$

on $\mathbb{T}$, where

(i) $\tau_{k} \in \mathbb{T}$ with $0 \leq t_{0}=\tau_{0}<\tau_{1}<\tau_{2}<\cdots$ and $\tau_{k} \rightarrow \infty$ as $k \rightarrow \infty$,

(ii) $f, g \in \mathrm{C}_{\mathrm{rd}}\left[\mathbb{T} \times \mathbb{R}^{n} \times \mathbb{R}^{n}, \mathbb{R}^{n}\right], z_{k} \in \mathbb{R}^{n}$ and $\lambda_{k}: \mathbb{T} \times \mathbb{R}^{n} \rightarrow \mathbb{R}^{n}$, $k=0,1,2, \ldots$.

REMARK 3.1. If $\mathbb{T}=\mathbb{N}$, then equations $(3.4)$ and (3.5) are equations (2.1) and (2.2) of [16]. In the case of $\mathbb{T}=\mathbb{R}$ they coincide with equations (2.1.1) and (2.2.2) in [7].

REMARK 3.2. The term "practical stability" is used to describe the ability of a time-delay system to be stable in the presence of small perturbations. Any equation governing the behavior of a perturbation is called a perturbed system. Sometimes, it may be convenient to involve perturbations in the definition of practical stability itself since one can then deal with perturbations directly as constraints [7].

By a solution $x(t)=x\left(t, t_{0}, x_{0}\right)$ of (3.4) we mean the following:

$$
x(t)= \begin{cases}x_{0}(t), & t_{0} \leq t \leq \tau_{1}, \\ x_{1}(t), & \tau_{1} \leq t \leq \tau_{2}, \\ \vdots & \\ x_{k}(t), & \tau_{k} \leq t \leq \tau_{k+1}, \\ \vdots & \end{cases}
$$

where $x_{k}(t)=x_{k}\left(t, \tau_{k}, z_{k}\right)$ is the solution of the dynamic system

$$
x_{k}^{\Delta}(t)=f\left(t, x_{k}(t), \lambda_{k}\left(\tau_{k}, z_{k}\right)\right), \quad x_{k}\left(\tau_{k}\right)=z_{k} \in \mathbb{R}^{n},
$$

for each $k=0,1,2, \ldots$, and $\tau_{k} \leq t \leq \tau_{k+1}$. The description of solutions of system 3.5 can be related to above. We assume that solutions of (3.4) and (3.5) exist and are unique for $t \geq t_{0}$.

We also need the scalar comparison hybrid dynamic system

$$
\begin{aligned}
& u^{\Delta}(t)=G\left(t, u, \delta_{k}\left(u_{k}\right)\right), \quad t \in\left[\tau_{k}, \tau_{k+1}\right], \\
& u\left(\tau_{k}\right)=v_{k} \in \mathbb{R}^{+},
\end{aligned}
$$


where $G \in \mathrm{C}_{\mathrm{rd}}\left[\mathbb{T} \times \mathbb{R}^{+} \times \mathbb{R}^{+}, \mathbb{R}\right]$ and $\delta_{k} \in \mathrm{C}\left[\mathbb{R}^{+}, \mathbb{R}^{+}\right]$. By a solution $r(t)=$ $r\left(t, t_{0}, u_{0}\right)$ of 3.7 , we mean the following:

$$
r(t)= \begin{cases}r_{0}(t), & t_{0} \leq t \leq \tau_{1}, \\ r_{1}(t), & \tau_{1} \leq t \leq \tau_{2}, \\ \vdots & \\ r_{k}(t), & \tau_{k} \leq t \leq \tau_{k+1}, \\ \vdots & \end{cases}
$$

where $r_{k}(t)=r_{k}\left(t, \tau_{k}, u_{k}\right)$ is the solution of

$$
u_{k}^{\Delta}=G\left(t, u_{k}(t), \delta_{k}\left(v_{k}\right)\right), \quad u_{k}\left(\tau_{k}\right)=v_{k} \in \mathbb{R}^{+},
$$

for each $k=0,1,2, \ldots$ and $\tau_{k} \leq t \leq \tau_{k+1}$.

We can now prove the required comparison result in terms of a Lyapunovlike function which is useful in discussing the qualitative behavior of solutions of (3.4) and (3.5).

Relating to system (3.1), we assume the following:

(H) The solution $x\left(t, t_{0}, x_{0}\right)$ of (3.1) is rd-continuous with respect to the initial data and $\left\|x\left(t, t_{0}, x_{0}\right)\right\|$ is locally Lipschitzian in $x_{0}$.

ThEOREM 3.1. Assume that condition $(\mathrm{H})$ holds and

(i) for $V \in \mathrm{C}_{\mathrm{rd}}\left[\mathbb{T} \times \mathbb{R}^{n}, \mathbb{R}^{+}\right], V(t, x)$ is locally Lipschitzian in $x$ for each right-dense $t \in \mathbb{T}$, and for $\tau_{j} \leq t \leq \tau_{j+1}, j=0,1,2, \ldots$,

$D_{+} V^{\Delta}\left(s, x\left(t, s, x_{s}\right)\right) \geq G\left(s, V\left(s, x\left(t, s, x_{s}\right)\right), \delta_{j}\left(V\left(\tau_{j}, x_{j}\right)\right)\right), \quad \tau_{j} \leq s \leq t$, where $G \in \mathrm{C}_{\mathrm{rd}}\left[\mathbb{T} \times \mathbb{R}^{+}, \mathbb{R}^{+}\right], G(t, u, v) \mu(t)+u$ is nondecreasing in $v$ for each $(t, u)$ and in $u$ for each $(t, v), x_{s}=x_{j}\left(s, \tau_{j}, x_{j}\right)$, and $\delta_{j}(v)$ is nondecreasing in $v$ for all $j$,

(ii) the minimal solution $r(t)=r\left(t, t_{0}, u_{0}\right)$ of 3.7 exists for $t \geq t_{0}$, $t \in \mathbb{T}$.

Then for any solution $y\left(t, t_{0}, x_{0}\right)$ of 3.5 we have

$$
V\left(t, y\left(t, t_{0}, x_{0}\right)\right) \geq r\left(t, t_{0}, V\left(t_{0}, x\left(t, t_{0}, x_{0}\right)\right)\right), \quad t \in \mathbb{T},
$$

provided $V\left(t_{0}, x\left(t, t_{0}, x_{0}\right)\right) \geq u_{0}$.

Proof. Let $y_{j}\left(t, \tau_{j}, x_{j}\right)$ be the solution of system 3.5 in the interval $\left[\tau_{j}, \tau_{j+1}\right]$ existing for $t \geq t_{0}, t \in \mathbb{T}$, where $x_{j}=y_{j-1}\left(\tau_{j}, \tau_{j-1}, x_{j-1}\right), j=$ $1,2, \ldots$. Set

$$
m(s)=V\left(s, x\left(t, s, x_{s}\right)\right), \quad \tau_{j} \leq s \leq t \leq \tau_{j+1},
$$

where $x_{s}=x_{j}\left(s, \tau_{j}, x_{j}\right)$. Then by condition (i), it is easy to derive the differential inequality

$$
D_{+} m^{\Delta}(s) \geq G\left(s, m(s), \delta_{r}\left(m_{r}\right)\right), \quad s \in\left[\tau_{j}, \tau_{j+1}\right],
$$

where $m_{j}=V\left(\tau_{j}, x_{j}\right)$. 
Consider the interval $\left[t_{0}, \tau_{1}\right]$, and set $m_{0}=m\left(t_{0}\right)=V\left(t_{0}, x\left(t, t_{0}, x_{0}\right)\right)$ $=u_{0}$. For $t_{0} \leq t \leq \tau_{1}, t_{0} \leq s \leq t$, we have

$$
D_{+} m^{\Delta}(s) \geq G\left(s, m(s), \delta_{0}\left(m_{0}\right)\right) .
$$

Hence, by Lemma 3.2 , we get

$$
V\left(t, y_{0}\left(t, t_{0}, x_{0}\right)\right) \geq r_{0}\left(t, t_{0}, V\left(t_{0}, x\left(t, t_{0}, x_{0}\right)\right)\right),
$$

where $r_{0}\left(t, t_{0}, u_{0}\right)$ is the minimal solution of

$$
u_{0}^{\Delta}(t)=G\left(t, u_{0}(t), \delta_{0}\left(u_{0}\right)\right), \quad u_{0}\left(t_{0}\right)=u_{0}, \quad t \in\left[t_{0}, \tau_{1}\right],
$$

and $y_{0}(t)=y_{0}\left(t, t_{0}, x_{0}\right)$ is the solution of

$$
y_{0}^{\Delta}(t)=g\left(t, y_{0}(t), \lambda_{0}\left(x_{0}\right)\right), \quad y_{0}\left(t_{0}\right)=x_{0}, \quad t \in\left[t_{0}, \tau_{1}\right] .
$$

Next, consider the interval $\left[\tau_{1}, \tau_{2}\right]$. Choosing $u_{1}=r_{0}\left(\tau_{1}, t_{0}, u_{0}\right)$ and $x_{1}=$ $y_{0}\left(\tau_{1}, t_{0}, x_{0}\right)$, we have

$$
D_{+} m^{\Delta}(s) \geq G\left(s, m(s), \delta_{1}\left(m_{1}\right)\right),
$$

where $m_{1}=V\left(\tau_{1}, y_{0}\left(\tau_{1}, t_{0}, x_{0}\right)\right) \geq r_{0}\left(\tau_{1}, t_{0}, u_{0}\right)=u_{1}$. In the interval $\left[\tau_{1}, \tau_{2}\right]$, using the monotonicity properties of $g(t, u, v)$ and $\delta_{1}(v)$ with respect to $v$ and assumption (i), we have

$$
D_{+} m^{\Delta}(s) \geq G\left(s, m(s), \delta_{1}\left(u_{1}\right)\right) .
$$

Again, by Lemma 3.2 with $u_{1}=V\left(\tau_{1}, x\left(t, \tau_{1}, x_{1}\right)\right)$, we have

$$
V\left(t, y_{1}\left(t, \tau_{1}, x_{1}\right)\right) \geq r_{1}\left(t, \tau_{1}, V\left(\tau_{1}, x\left(t, \tau_{1}, x_{1}\right)\right)\right),
$$

where $r_{1}\left(t, t_{1}, u_{1}\right)$ is the minimal solution of

$$
u_{1}^{\Delta}(t)=G\left(t, u_{1}(t), \delta_{1}\left(u_{1}\right)\right), \quad u_{1}\left(\tau_{1}\right)=u_{1}, \quad t \in\left[\tau_{1}, \tau_{2}\right],
$$

and $y_{1}(t)=y_{1}\left(t, \tau_{1}, x_{1}\right)$ is the solution of

$$
y_{1}^{\Delta}(t)=g\left(t, y_{1}(t), \lambda_{1}\left(x_{1}\right)\right), \quad y_{1}\left(\tau_{1}\right)=x_{1}, \quad t \in\left[\tau_{1}, \tau_{2}\right] .
$$

We repeat the process using the special choice of $x_{j}=y_{j-1}\left(\tau_{j}, \tau_{j-1}, x_{j-1}\right)$, $j=1,2, \ldots$, to get

$$
V\left(t, x_{j}(t)\right) \geq r_{j}\left(t, \tau_{j}, V\left(\tau_{j}, x\left(t, \tau_{j}, x_{j}\right)\right)\right),
$$

where $r_{j}\left(t, \tau_{j}, u_{j}\right)$ is the minimal solution of

$$
u_{j}^{\Delta}(t)=G\left(t, u_{j}(t), \delta_{j}\left(u_{j}\right)\right), \quad u_{j}\left(\tau_{j}\right)=u_{j}, \quad t \in\left[\tau_{j}, \tau_{j+1}\right],
$$

and $y_{j}(t)=y_{j}\left(t, \tau_{j}, x_{j}\right)$ is the solution of

$$
y_{j}^{\Delta}(t)=g\left(t, y_{j}(t), \lambda_{j}\left(x_{j}\right)\right), \quad y_{j}\left(\tau_{r}\right)=x_{j}, \quad t \in\left[\tau_{j}, \tau_{j+1}\right] .
$$


Defining for $t \in \mathbb{T}, t \geq t_{0}$,

$$
V(t, y(t))= \begin{cases}V\left(t, y_{0}(t)\right), & t_{0} \leq t \leq \tau_{1}, \\ V\left(t, y_{1}(t)\right), & \tau_{1} \leq t \leq \tau_{2}, \\ \vdots & \\ V\left(t, y_{j}(t)\right), & \tau_{j} \leq t \leq \tau_{j+1}, \\ \vdots & \end{cases}
$$

with $V\left(t, x\left(t, t_{0}, x_{0}\right)\right)=u_{0}$, we have the desired estimate 3.9 for $t \geq t_{0}$ and the proof is complete.

Estimate (3.9) shows a connection between solutions of systems 3.4 and (3.5) in terms of the minimal solution of (3.7). Similarly, we can give a connection between solutions of systems (3.4) and (3.5) in terms of the maximal solution of 3.7 .

THEOREM 3.2. Assume that condition $(\mathrm{H})$ holds and

(i) for $V \in \mathrm{C}_{\mathrm{rd}}\left[\mathbb{T} \times \mathbb{R}^{n}, \mathbb{R}^{+}\right], V(t, x)$ is locally Lipschitzian in $x$ for each right-dense $t \in \mathbb{T}$, and for $\tau_{j} \leq t \leq \tau_{j+1}, j=0,1,2, \ldots$,

$D^{+} V^{\Delta}\left(s, x\left(t, s, x_{k}\right)\right) \leq G\left(s, V\left(s, x\left(t, s, x_{s}\right)\right), \delta_{j}\left(V\left(\tau_{j}, x_{j}\right)\right)\right), \quad \tau_{j} \leq s \leq t$, where $G \in \mathrm{C}_{\mathrm{rd}}\left[\mathbb{T}^{k} \times \mathbb{R}^{+}, \mathbb{R}^{+}\right], G(t, u, v) \mu(t)+u$ is nondecreasing in $v$ for each $(t, u)$ and in $u$ for each $(t, v)$, and $\delta_{j}(v)$ is nondecreasing in $v$ for all $j$,

(ii) the maximal solution $\beta(t)=\beta\left(t, t_{0}, u_{0}\right)$ of 3.7 exists for $t \geq t_{0}$, $t \in \mathbb{T}$.

Then for any solution $y\left(t, t_{0}, x_{0}\right)$ of 3.5 we have

$$
V\left(t, y\left(t, t_{0}, x_{0}\right)\right) \leq \beta\left(t, t_{0}, V\left(t_{0}, x\left(t, t_{0}, x_{0}\right)\right)\right), \quad t \in \mathbb{T},
$$

provided $V\left(t_{0}, x\left(t, t_{0}, x_{0}\right)\right) \leq u_{0}$.

4. Practical stability in terms of two measures. Using the comparison Theorems 3.1 and 3.2 , we prove some results on practical stability and strict practical stability of system (3.5) in terms of two measures when information about systems $(3.4)$ and $(3.7)$ is known. For the sake of convenience, we introduce the following function classes:

$$
\begin{aligned}
& \mathcal{K}=\left\{\alpha \in \mathrm{C}\left[\mathbb{R}^{+}, \mathbb{R}^{+}\right]: \alpha \text { is strictly increasing and } \alpha(0)=0\right\} \\
& \Gamma=\left\{h \in \mathrm{C}_{\mathrm{rd}}\left[\mathbb{T} \times \mathbb{R}^{n}, \mathbb{R}^{+}\right]: \inf _{(t, x)} h(t, x)=0\right\} \\
& \Sigma=\left\{Q \in \mathrm{C}\left[\mathbb{R}^{+}, \mathbb{R}^{+}\right]: Q(0)=0 \text { and } Q \text { is increasing }\right\} \\
& S(h, \gamma)=\left\{(t, x) \in \mathbb{T} \times \mathbb{R}^{n}: h \in \Gamma \text { and } h(t, x)<\gamma, \gamma>0\right\}
\end{aligned}
$$


Definition 4.1. The hybrid dynamic system 3.4 is said to be

(i) practically stable if for given $0<\lambda<A$, the condition $\left\|x_{0}\right\|<\lambda$ implies $\|x(t)\|<A, t \geq t_{0}$ for some $t_{0} \in \mathbb{T}$, where $x(t)=x\left(t, t_{0}, x_{0}\right)$ is any solution of 3.4 ;

(ii) practically quasi-stable if for given $\lambda, B, T>0$ and some $t_{0} \in \mathbb{T}$ with $t_{0}+T \in \mathbb{T}$, the condition $\left\|x_{0}\right\|<\lambda$ implies $\|x(t)\|<B, t \geq t_{0}+T$, $t \in \mathbb{T}$;

(iii) strongly practically stable if (i) and (ii) hold simultaneously;

(iv) practically asymptotically stable if (i) holds and for any given $\varepsilon>0$ there exists $T_{0}>0$ such that $t_{0}+T_{0} \in \mathbb{T}$ and $\left\|x_{0}\right\|<\lambda$ implies $\|x(t)\|<\varepsilon, t \geq t_{0}+T_{0}$

(v) strictly practicallly stable if there exist $0<\lambda_{1} \leq A_{1}$ such that $\left\|x_{0}\right\|<$ $\lambda_{1}$ implies $\|x(t)\|<A_{1}, t \geq t_{0}$, for some $t_{0} \in \mathbb{T}$, and for every $0<\lambda_{2} \leq \lambda_{1}$ there exists $A_{2} \leq \lambda_{2}$ such that $\left\|x_{0}\right\|>\lambda_{2}$ implies $\|x(t)\|>A_{2}, t \geq t_{0}$.

Definition 4.2. Let $h, h_{0} \in \Gamma$. The hybrid dynamic system 3.4 is said to be

(PS1) $\left(h_{0}, h\right)$-practically stable if for given $0<\lambda<A$, the condition $h_{0}\left(t_{0}, x_{0}\right)<\lambda$ implies $h(t, x(t))<A, t \geq t_{0}$, for some $t_{0} \in \mathbb{T}$, where $x(t)=x\left(t, t_{0}, x_{0}\right)$ is any solution of $(3.4)$;

(PS2) $\left(h_{0}, h\right)$-practically quasi-stable if for given $\lambda, B, T>0$ and some $t_{0} \in \mathbb{T}$ with $t_{0}+T \in \mathbb{T}$, the condition $h_{0}\left(t_{0}, x_{0}\right)<\lambda$ implies $h(t, x(t))<B, t \geq t_{0}+T, t \in \mathbb{T}$

(PS3) $\left(h_{0}, h\right)$-strongly practically stable if (PS1) and (PS2) hold simultaneously;

(PS4) $\left(h_{0}, h\right)$-practically asymptotically stable if (PS1) holds and for any given $\varepsilon>0$ there exists $T_{0}>0$ such that $t_{0}+T_{0} \in \mathbb{T}$ and $h_{0}\left(t_{0}, x_{0}\right)<\lambda$ implies $h(t, x(t))<\varepsilon, t \geq t_{0}+T_{0}$;

(PS5) $\left(h_{0}, h\right)$-strictly practically stable if there exist $0<\lambda_{1} \leq A_{1}$ such that $h_{0}\left(t_{0}, x_{0}\right)<\lambda_{1}$ implies $h(t, x(t))<A_{1}, t \geq t_{0}$, for some $t_{0} \in \mathbb{T}$, and for every $0<\lambda_{2} \leq \lambda_{1}$ there exists $A_{2} \leq \lambda_{2}$ such that $h_{0}\left(t_{0}, x_{0}\right)>\lambda_{2}$ implies $h(t, x(t))>A_{2}, t \geq t_{0}$.

One can similarly define corresponding notions for the scalar comparison dynamic system (3.7).

Definition 4.3. Let $Q_{0}, Q \in \Sigma$. Then we say that the scalar comparison hybrid dynamic system 3.7) is $\left(Q_{0}, Q\right)$-practically stable if for given $0<\lambda$ $<A$, the condition $Q_{0}\left(t_{0}, u_{0}\right)<\lambda$ implies $Q(t, u(t))<A, t \geq t_{0}, t \in \mathbb{T}$, where $u(t)=u\left(t, t_{0}, u_{0}\right)$ is any solution of 3.7 .

Other practical stability notions can be defined similarly. 
THEOREM 4.1. Assume condition $(\mathrm{H})$ holds and

(A1) $0<\lambda<A$;

(A2) $h_{0}, h^{*}, h \in \Gamma, h^{*}(t, x)$ is nondecreasing in $t$ and $h_{0}$ is uniformly finer than $h$, i.e.,

$$
h(t, x) \leq \phi\left(h_{0}(t, x)\right), \phi \in \mathcal{K}, \quad \text { whenever } h_{0}(t, x)<\lambda ;
$$

(A3) there exists $V \in \mathrm{C}_{\mathrm{rd}}\left[\mathbb{T} \times \mathbb{R}^{n}, \mathbb{R}^{+}\right]$such that $V(t, u)$ is locally Lipschitzian in $u$ for each right-dense $t \in \mathbb{T}$, and for $a, b \in \mathcal{K}$,

$$
\begin{array}{ll}
V(t, x) \geq b(h(t, x)) & \text { if }(t, x) \in S(h, A), \\
V(t, x) \leq a\left(h^{*}(t, x)\right) & \text { if }(t, x) \in S\left(h^{*}, \lambda\right) ;
\end{array}
$$

(A4) for $(t, x) \in S(h, A)$,

$$
D^{+} V^{\Delta}\left(s, x\left(t, s, x_{k}\right)\right) \leq G\left(s, V\left(s, x\left(t, s, x_{k}\right)\right), \delta_{r}\left(V\left(\tau_{j}, x_{j}\right)\right)\right),
$$

where $G \in \mathrm{C}_{\mathrm{rd}}\left[\mathbb{T}^{k} \times \mathbb{R}^{+} \times \mathbb{R}^{+}, \mathbb{R}^{+}\right], G(t, u, v) \mu(t)+u$ is nondecreasing in $v$ for each $(t, u)$ and in $u$ for each $(t, v)$, and $\delta_{j}(v)$ is nondecreasing in $v$ for all $j$;

(A5) $\phi(\lambda)<A$ and $a(\lambda)<b(A)$;

(A6) system (3.4) is $\left(h_{0}, h^{*}\right)$-practically stable with respect to $(\lambda, \lambda)$, i.e.,

$$
h_{0}\left(t_{0}, x_{0}\right)<\lambda \quad \text { implies } h^{*}\left(t, x\left(t, t_{0}, x_{0}\right)\right)<\lambda, t \geq t_{0} .
$$

Then practical stability properties of system (3.7) imply the corresponding $\left(h_{0}, h\right)$-practical stability properties of the perturbed system 3.5.

Proof. Assume that (3.7) is practically stable. Then for given $(a(\lambda), b(A))$,

$$
u_{0}<a(\lambda) \text { implies } u\left(t, t_{0}, u_{0}\right)<b(A), \quad t \geq t_{0},
$$

where $u\left(t, t_{0}, u_{0}\right)$ is any solution of (3.7). Since (3.4) is $\left(h_{0}, h^{*}\right)$-practically stable with respect to $(\lambda, \lambda), 4.1$ holds. Then by $(\mathrm{A} 2)$ and $(\mathrm{A} 5)$, it follows that

$$
h\left(t_{0}, x_{0}\right) \leq \phi\left(h_{0}\left(t_{0}, x_{0}\right)\right)<\phi(\lambda)<A .
$$

We claim that $h(t, y(t))<A, t \geq t_{0}$, where $y(t)=y\left(t, t_{0}, x_{0}\right)$ is any solution of 3.5 . Indeed, if this were not true, there would exist a solution $y\left(t, t_{0}, x_{0}\right)$ of (3.5) with $h_{0}\left(t_{0}, x_{0}\right)<\lambda$ and a $t_{1}>t_{0}, t_{1} \in \mathbb{T}$, such that

$$
h\left(t_{1}, y\left(t_{1}, t_{0}, x_{0}\right)\right) \geq A, \quad h\left(t, y\left(t, t_{0}, x_{0}\right)\right)<A, \quad t_{0} \leq t<t_{1} .
$$

As $h_{0}\left(t_{0}, x_{0}\right)<\lambda$, Theorem 3.2 together with (A2), (A3) and 4.3) imply

$$
V\left(t, y\left(t, t_{0}, x_{0}\right)\right) \leq \beta\left(t, t_{0}, V\left(t_{0}, x\left(t, t_{0}, x_{0}\right)\right)\right), \quad t_{0} \leq t \leq t_{1},
$$

and

$$
V\left(t_{0}, x\left(t_{1}, t_{0}, x_{0}\right)\right) \leq a\left(h^{*}\left(t_{0}, x\left(t_{1}, t_{0}, x_{0}\right)\right)\right) \leq a\left(h^{*}\left(t_{1}, x\left(t_{1}, t_{0}, x_{0}\right)\right)\right)<a(\lambda) .
$$


Using (A3), 4.1), 4.3) and 4.4), we have

$$
\begin{aligned}
b(A) & \leq b\left(h\left(t_{1}, y\left(t_{1}, t_{0}, x_{0}\right)\right)\right) \leq V\left(t_{1}, y\left(t_{1}, t_{0}, x_{0}\right)\right) \\
& \leq \beta\left(t_{1}, t_{0}, V\left(t_{0}, x\left(t_{1}, t_{0}, x_{0}\right)\right)\right)<b(A),
\end{aligned}
$$

and this contradiction proves that $h_{0}\left(t_{0}, x_{0}\right)<\lambda$ implies $h\left(t, y\left(t, t_{0}, x_{0}\right)\right)<A$, $t \geq t_{0}$.

Next, we prove that system (3.5) is $\left(h_{0}, h\right)$-strongly practically stable for given positive numbers $\lambda, A, B, T$. To do this, suppose that (3.7) is strongly practically stable for positive numbers $a(\lambda), b(A), b(B), T$. This means we only need to prove $\left(h_{0}, h\right)$-practical quasi-stability of system (3.5). Practical quasi-stability of (3.7) means that

$$
u_{0}<a(\lambda) \text { implies } u\left(t, t_{0}, u_{0}\right)<b(B), t \geq t_{0}+T, \quad \text { with } t_{0}+T \in \mathbb{T} .
$$

From the foregoing argument, since $V\left(t_{0}, x\left(t, t_{0}, x_{0}\right)\right)<a(\lambda)$ if $h_{0}\left(t_{0}, x_{0}\right)<\lambda$, we have

$$
b\left(h\left(t, y\left(t, t_{0}, x_{0}\right)\right)\right) \leq V\left(t, y\left(t, t_{0}, x_{0}\right)\right) \leq u\left(t, t_{0}, V\left(t_{0}, x\left(t, t_{0}, x_{0}\right)\right)\right)<b(B)
$$

for all $t \geq t_{0}+T$ if $h_{0}\left(t_{0}, x_{0}\right)<\lambda$. Thus we have $h(t, x(t))<B, t \geq t_{0}+T$, provided $h_{0}\left(t_{0}, x_{0}\right)<\lambda$. Hence system (3.5) is $\left(h_{0}, h\right)$-strongly practically stable.

Finally, we show that system 3.5$)$ is $\left(h_{0}, h\right)$-practically asymptotically stable. Now, let us suppose that (3.7) is practically asymptotically stable. This implies we only need to prove that for any given $\varepsilon>0$ there exists $T_{0}>0$ with $t_{0}+T_{0} \in \mathbb{T}$ such that $t_{0}+T_{0} \geq T$ and $h_{0}\left(t_{0}, x_{0}\right)<\lambda$ implies $h(t, x(t))<B, t \geq t_{0}+T_{0}$, for system (3.5). Practical asymptotic stability of (3.7) means that

$$
u_{0}<a(\lambda) \text { implies } u\left(t, t_{0}, u_{0}\right)<b(B), t \geq t_{0}+T_{0} .
$$

From the argument above, since $V\left(t_{0}, x\left(t, t_{0}, x_{0}\right)\right)<a(\lambda)$ whenever $h_{0}\left(t_{0}, x_{0}\right)$ $<\lambda$, we obtain

$$
b\left(h\left(t, y\left(t, t_{0}, x_{0}\right)\right)\right) \leq V\left(t, y\left(t, t_{0}, x_{0}\right)\right) \leq u\left(t, t_{0}, V\left(t_{0}, x\left(t, t_{0}, x_{0}\right)\right)\right)<b(B)
$$

for all $t \geq t_{0}+T_{0}$ if $h_{0}\left(t_{0}, x_{0}\right)<\lambda$. Thus we have $h(t, x(t))<B, t \geq t_{0}+T_{0}$ provided $h_{0}\left(t_{0}, x_{0}\right)<\lambda$. Hence system (3.5) is $\left(h_{0}, h\right)$-strongly practically stable, and the proof is complete.

THEOREM 4.2. Suppose that conditions of Theorem 4.1 are satisfied except that condition (A3) is replaced by

(A7) $Q_{0}, Q \in \Sigma$, and for $a, b \in \mathcal{K}$

$$
\begin{aligned}
Q(V(t, x)) & \geq b(h(t, x)) & & \text { if }(t, x) \in S(h, A), \\
Q_{0}(V(t, x)) & \leq a\left(h^{*}(t, x)\right) & & \text { if }(t, x) \in S\left(h^{*}, \lambda\right) .
\end{aligned}
$$

Then $\left(Q_{0}, Q\right)$-practical stability properties of system (3.7) imply the corresponding $\left(h_{0}, h\right)$-practical stability properties of the perturbed system (3.5). 
Proof. Assume that 3.7 is $\left(Q_{0}, Q\right)$-practically stable. Then we have $(a(\lambda), b(A))$ such that

$$
Q_{0}\left(u_{0}\right)<a(\lambda) \text { implies } Q\left(u\left(t, t_{0}, u_{0}\right)\right)<b(A), t \geq t_{0} .
$$

Suppose that the $\left(h_{0}, h\right)$-practical stability of $(3.5)$ does not hold. Then arguing as in Theorem 4.1, by (A2), (A7) and 4.3) we can show that

$$
\begin{aligned}
Q_{0}\left(V\left(t_{0}, x\left(t_{1}, t_{0}, x_{0}\right)\right)\right) & \leq a\left(h^{*}\left(t_{0}, x\left(t_{1}, t_{0}, x_{0}\right)\right)\right) \\
& \leq a\left(h^{*}\left(t_{1}, x\left(t_{1}, t_{0}, x_{0}\right)\right)\right)<a(\lambda) .
\end{aligned}
$$

Then using (A7), 4.1), 4.3) and 4.4), we have

$$
\begin{aligned}
b(A) & \leq b\left(h\left(t_{1}, y\left(t_{1}, t_{0}, x_{0}\right)\right)\right) \leq Q\left(V\left(t_{1}, y\left(t_{1}, t_{0}, x_{0}\right)\right)\right) \\
& \leq Q\left(u\left(t_{1}, t_{0}, V\left(t_{0}, x\left(t_{1}, t_{0}, x_{0}\right)\right)\right)\right)<b(A),
\end{aligned}
$$

which is a contradiction. The proof is complete.

REMARK 4.1. If $h_{0}=h^{*}=h=\|x\|$, then we get the usual practical stability of system 3.5.

REMARK 4.2. If $Q_{0}(u)=Q(u)=u$, we deduce Theorem 4.1 .

The following result concerns $\left(h_{0}, h\right)$-strict practical stability of (3.5).

THEOREM 4.3. Assume that condition $(\mathrm{H})$ holds and

(B1) $h_{0}, h^{*}, h \in \Gamma, h^{*}(t, x)$ is nondecreasing in $t$ and $h_{0}$ is uniformly finer than $h$, i.e., $h(t, x) \leq \phi\left(h_{0}(t, x)\right), \phi \in \mathcal{K}$, whenever $h_{0}(t, x)<\lambda$, and $h(t, x) \geq \phi\left(h_{0}(t, x)\right), \phi \in \mathcal{K}$, whenever $h_{0}(t, x)>\lambda$;

(B2) for each $0<\eta \leq A_{i}$, there exist $V_{i} \in \mathrm{C}_{\mathrm{rd}}\left[\mathbb{T} \times \mathbb{R}^{n}, \mathbb{R}^{+}\right]$such that $V_{i}(t, x)$ is locally Lipschitzian in $x$ for each right-dense $t \in \mathbb{T}$, and there exist $a_{i}, b_{i} \in \mathcal{K}, i=1,2$, such that

$$
\begin{array}{ll}
V_{1}(t, x) \geq b_{1}(h(t, x)) & \text { if } h(t, x) \leq \eta, \\
V_{1}(t, x) \leq a_{1}\left(h^{*}(t, x)\right) & \text { if } h^{*}(t, x) \leq \eta,
\end{array}
$$

and

$$
\begin{array}{ll}
V_{2}(t, x) \leq a_{2}(h(t, x)) & \text { if } h(t, x) \geq \eta, \\
V_{2}(t, x) \geq b_{2}\left(h^{*}(t, x)\right) & \text { if } h^{*}(t, x) \geq \eta ;
\end{array}
$$

(B3)

$$
\begin{gathered}
D^{+} V_{1}^{\Delta}\left(s, x\left(t, s, x_{k}\right)\right) \leq G\left(s, V\left(s, x\left(t, s, x_{k}\right)\right), \delta_{r}\left(V\left(\tau_{r}, x_{r}\right)\right)\right) \\
\text { for } h(t, x) \leq A_{1}, \\
D_{+} V_{2}^{\Delta}\left(s, x\left(t, s, x_{k}\right)\right) \geq G\left(s, V\left(s, x\left(t, s, x_{k}\right)\right), \delta_{r}\left(V\left(\tau_{r}, x_{r}\right)\right)\right) \\
\text { for } h(t, x) \geq A_{2},
\end{gathered}
$$


where $G \in C_{\mathrm{rd}}\left[\mathbb{T}^{k} \times \mathbb{R}^{+} \times \mathbb{R}^{+}, \mathbb{R}^{+}\right], G(t, u, v) \mu(t)+u$ is nondecreasing in $v$ for each $(t, u)$ and in $u$ for each $(t, v)$, and $\delta_{r}(v)$ is nondecreasing in $v$ for all $r$;

(B4) $\phi\left(\lambda_{1}\right)<A_{1}, \phi\left(\lambda_{2}\right)>A_{2}, a_{1}\left(\lambda_{1}\right)<b_{1}\left(A_{1}\right)$ and $a_{2}\left(A_{2}\right)<b_{2}\left(\lambda_{2}\right)$;

(B5) system (3.4) is $\left(h_{0}, h^{*}\right)$-strictly practically stable with respect to $\left(\lambda_{1}, \lambda_{1}\right)$ and $\left(\lambda_{2}, \lambda_{2}\right)$, i.e., $h_{0}\left(t_{0}, x_{0}\right)<\lambda_{1}$ implies $h^{*}\left(t, x\left(t, t_{0}, x_{0}\right)\right)$ $<\lambda_{1}$ and $h_{0}\left(t_{0}, x_{0}\right)>\lambda_{2}$ implies $h^{*}\left(t, x\left(t, t_{0}, x_{0}\right)\right)>\lambda_{2}, t \geq t_{0}$.

Then strict practical stability properties of system (3.7) imply the corresponding strict $\left(h_{0}, h\right)$-practical stability properties of the perturbed system (3.5).

Proof. Assume that (3.7) is strictly practically stable. Then there exist $0<\lambda_{1} \leq A_{1}$ such that $a_{1}\left(\lambda_{1}\right)<b_{1}\left(A_{1}\right)$ and $u_{0}<a_{1}\left(\lambda_{1}\right)$ implies $u(t)<$ $b_{1}\left(A_{1}\right), t \geq t_{0}$, for some $t_{0} \in \mathbb{T}$, and for every $0<\lambda_{2} \leq \lambda_{1}$ there exists $A_{2} \leq \lambda_{2}$ such that $v_{0}>b_{2}\left(\lambda_{2}\right)$ implies $v(t)>a_{2}\left(A_{2}\right), t \geq t_{0}$, where $u(t)=$ $u\left(t, t_{0}, u_{0}\right)$ and $v(t)=v\left(t, t_{0}, u_{0}\right)$ are maximal and minimal solutions of (3.7), respectively.

Since (3.4) is $\left(h_{0}, h^{*}\right)$-strictly practically stable with respect to $\left(\lambda_{1}, \lambda_{1}\right)$ and $\left(\lambda_{2}, \lambda_{2}\right)$, it follows that

$$
\begin{array}{llll}
h_{0}\left(t_{0}, x_{0}\right)<\lambda_{1} & \text { implies } & h^{*}\left(t, x\left(t, t_{0}, x_{0}\right)\right)<\lambda_{1}, & t \geq t_{0}, \\
h_{0}\left(t_{0}, x_{0}\right)>\lambda_{2} & \text { implies } & h^{*}\left(t, x\left(t, t_{0}, x_{0}\right)\right)>\lambda_{2}, & t \geq t_{0} .
\end{array}
$$

Then by (B1) and (B4),

$$
\begin{array}{llll}
h\left(t_{0}, x_{0}\right) \leq \phi\left(h_{0}\left(t_{0}, x_{0}\right)\right)<\phi\left(\lambda_{1}\right)<A_{1} & \text { whenever } & h_{0}\left(t_{0}, x_{0}\right)<\lambda_{1}, \\
h\left(t_{0}, x_{0}\right) \geq \phi\left(h_{0}\left(t_{0}, x_{0}\right)\right)>\phi\left(\lambda_{2}\right)>A_{2} & \text { whenever } & h_{0}\left(t_{0}, x_{0}\right)>\lambda_{2} .
\end{array}
$$

Now we claim that $h_{0}\left(t_{0}, x_{0}\right)<\lambda_{1}$ implies $h(t, y(t))<A_{1}, t \geq t_{0}$, where $y(t)=y\left(t, t_{0}, x_{0}\right)$ is any solution of $(3.5)$. If this were not true, there would exist a solution $y\left(t, t_{0}, x_{0}\right)$ of 3.5 with $h_{0}\left(t_{0}, x_{0}\right)<\lambda_{1}$ and a $t_{1} \in \mathbb{T}, t_{1}>t_{0}$, such that

$$
h\left(t_{1}, y\left(t_{1}, t_{0}, x_{0}\right)\right) \geq A_{1}, \quad h\left(t, y\left(t, t_{0}, x_{0}\right)\right)<A_{1}, \quad t_{0} \leq t<t_{1} .
$$

Then by Theorem 3.2 , we obtain

$$
V_{1}\left(t, y\left(t, t_{0}, x_{0}\right)\right) \leq u_{1}\left(t, t_{0}, V_{1}\left(t_{0}, x\left(t, t_{0}, x_{0}\right)\right)\right), \quad t_{0} \leq t \leq t_{1} .
$$

Because of (B1), (B2) and 4.6), we have

$$
\begin{aligned}
V_{1}\left(t_{0}, x\left(t_{1}, t_{0}, x_{0}\right)\right) & \leq a_{1}\left(h^{*}\left(t_{0}, x\left(t_{1}, t_{0}, x_{0}\right)\right)\right) \\
& \leq a_{1}\left(h^{*}\left(t_{1}, x\left(t_{1}, t_{0}, x_{0}\right)\right)\right)<a_{1}\left(\lambda_{1}\right)
\end{aligned}
$$

for $h_{0}\left(t_{0}, x_{0}\right)<\lambda_{1}$. Using (B2), (4.8) and (4.9), we have

$$
\begin{aligned}
b_{1}\left(A_{1}\right) & \leq b_{1}\left(h\left(t_{1}, y\left(t_{1}, t_{0}, x_{0}\right)\right)\right) \leq V_{1}\left(t_{1}, y\left(t_{1}, t_{0}, x_{0}\right)\right) \\
& \leq u_{1}\left(t_{1}, t_{0}, V_{1}\left(t_{0}, x\left(t_{1}, t_{0}, x_{0}\right)\right)\right)<b\left(A_{1}\right),
\end{aligned}
$$


and this contradiction proves that $h_{0}\left(t_{0}, x_{0}\right)<\lambda_{1}$ implies $h\left(t, y\left(t, t_{0}, x_{0}\right)\right)$ $<A_{1}, t \geq t_{0}$.

On the other hand, for every $0<\lambda_{2} \leq \lambda_{1}$, we claim that $h_{0}\left(t_{0}, x_{0}\right)>\lambda_{2}$ implies $h(t, y(t))>A_{2}, t \geq t_{0}$. If this were not true, there would exist a solution $y\left(t, t_{0}, x_{0}\right)$ of 3.5 with $h_{0}\left(t_{0}, x_{0}\right)>\lambda_{2}$ and a $t_{1} \in \mathbb{T}$ with $t_{1}>t_{0}$ such that

$$
\begin{array}{ll}
h(t, y(t)) \leq A_{2} & \text { for } t \geq t_{1}, \\
A_{2} \leq h(t, y(t)) \leq A_{1} & \text { for } t_{0} \leq t<t_{1} .
\end{array}
$$

Then by Theorem 3.1, we get

$$
V_{2}\left(t, y\left(t, t_{0}, x_{0}\right)\right) \geq u_{2}\left(t, t_{0}, V_{2}\left(t_{0}, x\left(t, t_{0}, x_{0}\right)\right)\right), \quad t_{0} \leq t \leq t_{1} .
$$

By (B1), (B2) and (3.7), we have

$$
V_{2}\left(t_{0}, x\left(t_{1}, t_{0}, x_{0}\right)\right) \geq b_{2}\left(h^{*}\left(t_{0}, x\left(t_{1}, t_{0}, x_{0}\right)\right)\right)>b_{2}\left(\lambda_{2}\right)
$$

for $h_{0}\left(t_{0}, x_{0}\right)>\lambda_{2}$. Using (B2), 4.10) and 4.11), we have

$$
\begin{aligned}
a_{2}\left(A_{2}\right) & \geq a_{2}\left(h\left(t_{1}, y\left(t_{1}, t_{0}, x_{0}\right)\right)\right) \geq V_{2}\left(t_{1}, y\left(t_{1}, t_{0}, x_{0}\right)\right) \\
& \geq u_{2}\left(t_{1}, t_{0}, V_{2}\left(t_{0}, x\left(t_{1}, t_{0}, x_{0}\right)\right)\right)>a_{2}\left(A_{2}\right),
\end{aligned}
$$

which is a contradiction. Hence system 3.5 is $\left(h_{0}, h\right)$-strictly practically stable, and the proof is complete.

THEOREM 4.4. Suppose that the conditions of Theorem 4.3 are satisfied except that the condition (B2) is replaced by

(B6) for any $0<\eta \leq A_{i}$, there exist $V_{i} \in \mathrm{C}_{\mathrm{rd}}\left[\mathbb{T} \times \mathbb{R}^{n}, \mathbb{R}^{+}\right]$such that $V_{i}(t, x)$ is locally Lipschitzian in $x$ for each right-dense $t \in \mathbb{T}$, and there exist $Q_{0}, Q \in \Sigma$ and $a_{i}, b_{i} \in \mathcal{K}, i=1,2$, such that

$$
\begin{array}{clrl}
Q\left(V_{1}(t, x)\right) & \geq b_{1}(h(t, x)) & & \text { if } h(t, x) \leq \eta, \\
Q_{0}\left(V_{1}(t, x)\right) & \leq a_{1}\left(h^{*}(t, x)\right) & & \text { if } h^{*}(t, x) \leq \eta
\end{array}
$$

and

$$
\begin{array}{cl}
Q\left(V_{2}(t, x)\right) \leq a_{2}(h(t, x)) & \text { if } h(t, x) \geq \eta, \\
Q_{0}\left(V_{2}(t, x)\right) \geq b_{2}\left(h^{*}(t, x)\right) & \text { if } h^{*}(t, x) \geq \eta .
\end{array}
$$

Then $\left(Q_{0}, Q\right)$-strict practical stability properties of system (3.7) imply the corresponding $\left(h_{0}, h\right)$-strict practical stability properties of the perturbed system (3.5).

The proof of Theorem 4.4 is similar to the proof of Theorem 4.2 and hence is omitted.

REMARK 4.3. If $\mathbb{T}=\mathbb{R}$, then Theorems 3.2, 4.1 and 4.2 of this paper coincide with Theorems 2.1, 3.1 and 3.2 in [16]. 
Acknowledgements. The authors wish to thank the referees for their valuable suggestions and useful comments.

This research is supported by the Natural Science Foundation of China (60774004, 60904024), China Postdoctoral Science Foundation Funded Project (20080441126, 200902564), Shandong Postdoctoral Foundation (200802018), the Natural Science Foundation of Shandong (Y2008A28, ZR2009AL003), and the Fund of Doctoral Program Research of the University of Jinan (XBS0843).

\section{References}

[1] D. Acheson, From Calculus to Chaos: An Introduction to Dynamics, Oxford Univ. Press, Oxford, 1997.

[2] M. Bohner and A. Peterson, Dynamic Equations on Time Scales, An Introduction with Applications, Birkhäuser, Boston, 2001.

[3] -, -, Advances in Dynamic Equations on Time Scales, Birkhäuser, Boston, 2003.

[4] F. H. Clark, Yu. S. Ledyaev, R. I. Stern and P. R. Woleski, Nonsmooth Analysis and Control Theory, Springer, New York, 1998.

[5] L. T. Grujić, Exact determination of a Lyapunov function and the asymptotic stability domain, Int. J. Systems Sci. 23 (1992), 1871-1888.

[6] S. Hilger, Analysis on measure chains - a unified approach to continuous and discrete calculus, Results Math. 18 (1990), 18-56.

[7] V. Lakshmikantham, S. Leela and A. A. Martynyuk, Practical Stability of Nonlinear Systems, World Sci., Singapore, 1990.

[8] V. Lakshmikantham and R. N. Mohapatra, Strict stability of differential equations, Nonlinear Anal. 46 (2001), 915-921.

[9] V. Lakshmikantham, S. Sivasundaram and B. Kaymakcalan, Dynamic Systems on Measure Chains, Kluwer, Dordrecht, 1996.

[10] V. Lakshmikantham and A. S. Vatsala, Hybrid systems on time scales, J. Comput. Appl. Math. 141 (2002), 227-235.

[11] V. Lakshmikantham and Y. Zhang, Strict practical stability of delay differential equation, Appl. Math. Comput. 118 (2001), 275-285.

[12] J. LaSalle and S. Lefschetz, Stability by Liapunov's Direct Method, Academic Press, New York, 1961.

[13] A. M. Nerode and W. Kohn, Models for Hybrid Systems, Lecture Notes in Comput. Sci. 36, Springer, Berlin, 1993.

[14] P. G. Wang and X. Liu, New comparison principle and stability criteria for impulsive hybrid systems on time scales, Nonlinear Anal. Real World Appl. 7 (2006), 10961103.

[15] - - - Practical stability of impulsive hybrid differential systems in terms of two measures on time scales, Nonlinear Anal. 65 (2006), 2035-2042.

[16] P. G. Wang, M. Wu and Y. H. Wu, Practical stability in terms of two measures for discrete hybrid systems, Nonlinear Anal. Hybrid Systems 2 (2008), 58-64. 
Shurong Sun

School of Science

University of Jinan

Jinan, Shandong 250022, P.R. China

and

Department of Mathematics and Statistics

Missouri University of Science

and Technology

Rolla, MO 65409-0020, U.S.A.

E-mail: sshrong@163.com

Elvan Akin-Bohner

Department of Mathematics and Statistics

Missouri University of Science

and Technology

Rolla, MO 65409-0020, U.S.A.

E-mail: akine@mst.edu
Zhenlai Han

School of Science

University of Jinan

Jinan, Shandong 250022, P.R. China

and

School of Control Science and Engineering

Shandong University

Jinan, Shandong 250061, P.R. China

E-mail: hanzhenlai@163.com

Ping Zhao

School of Control Science and Engineering

University of Jinan

Jinan, Shandong 250022, P.R. China

E-mail: cse_zhaop@ujn.edu.cn

Received June 6, 2010;

received in final form December 9, 2010 
